\title{
Lachgas: guter analgetisch-anxiolytischer Effekt
}

- Äquimolare analgetische Fertigmischungen aus Sauerstoff und Lachgas $\left(\mathrm{N}_{2} \mathrm{O}\right)$ sind in vielen europäischen Ländern seit Jahrzehnten etablierter Standard. In Deutschland erst seit 2008 unter dem Namen LIVOPAN $^{\circledR}$ zugelassen, zeigen Erfahrungen aus pädiatrischen Zentren, dass die fixe Kom- bination aus dem klinischen Alltag nicht mehr wegzudenken ist. Zugelassen für die Behandlung kurzzeitiger Schmerzzustände leichter bis mittlerer Intensität, kommt das Gemisch vor allem in der Kinderchirurgie bei kleineren Eingriffen zum Einsatz und kann nach entsprechender Schulung auch von

\section{Biologika kommen immer häufiger zum Einsatz}

Eine frühe Diagnose und Behandlung sind entscheidend für den Krankheitsverlauf von Patienten mit früher rheumatoider Arthritis (RA). Damit Patienten zeitig vorselektioniert und einem Rheumatologen zugeführt werden können, sollte die Bestimmung der Antikörper gegen CCP (Cyclisches Citrulliniertes Peptid) zunehmend bereits durch den Hausarzt erfolgen, riet Prof. Dr. Markus Gaubitz, Münster. Er betonte zudem, dass es immer mehr Daten gäbe, die dafür sprechen, eine Behandlung mit DMARD (Disease Modifying Anti-Rheumatic Drug) bereits im Stadium der undifferenzierten RA, also bei anti-CCPAntikörper-negativen Patienten mit entsprechender Klinik sowie Entzündungszeichen (BSG, CRP, RF), zu beginnen. Neu ist zudem die Einschätzung, dass bei schlechten Prognosefaktoren nach heutigem Verständnis bereits nach Versagen des ersten traditionellen DMARD Biologika eingesetzt werden sollten. „Der frühe Einsatz von Biologika ist nicht nur klinisch effektiv, sondern langfristig auch kosteneffektiv," sagte Gaubitz.

Auch bei Kindern mit polyartikulärer juveniler idiopathischer Arthritis (JIA) werden Biologika immer früher eingesetzt, wie die Auswertung des BIKER(Biologika in der Kinderrheumatologie)-Registers zeigt. Für die Therapie in ganz jungen Jahren, d.h. ab einem Alter von zwei Jahren, ist seit September diesen Jahres Etanercept (Enbrel ${ }^{\circledR}$ ) zugelassen, wie Prof. Dr. Gerd Horneff, Sankt Augustin, sagte. Anders als für viele andere Medikamente in der Kinderrheumatologie sind Langzeitwirksamkeit und -sicherheit durch Registerdaten gut untersucht. Laut Horneff können zwar mögliche Risiken in Form von Infektionen, Autoimmunopathien und Malignomen nicht vollkommen ausgeschlossen werden. „Ernsthafte Infektionen sind jedoch zählbar," so Horneff. „Ihre Rate hängt primär von der Begleitmedikation, vor allem dem Einsatz von Steroiden, ab."

Dr. Wiebke Kathmann

Symposium "Systemische entzündlich-rheumatische Erkrankungen in Gegenwart und Zukunft". 39. Kongress der DGRh. München, 2. September 2011. Veranstalter: Pfizer

\section{Kopflausmittel wirkt trotz Genmutation}

— Endlich besteht Klarheit: Selbst Infestationen mit resistenten Kopfläusen können mit den bewährten Arzneimitteln wirksam behandelt werden. So lauten die Ergebnisse aktueller Studien des Universitätsklinikums SchleswigHolstein in Kiel. Die Mittel enthalten behördlich geprüfte und anerkannte Wirkstoffe wie Pyrethrumextrakt bzw. von Pyrethrum abgeleitete synthetische Pedikulozide. Im Rahmen der Studie wurde der Behandlungserfolg bei Kindern untersucht, auf deren Köpfen resistente Läuse molekularbiologisch nachgewiesen werden konnten. Obwohl $93 \%$ der Läuse nicht-ärztlichem Personal angewendet werden. Bei bedarfsgerechter Einatmung des Gases über eine Maske ist die Wirkung gut regulierbar: Die Schmerzlinderung ist rasch und effektiv, nach Ende der Inhalation klingt sie schnell ab. Die Kinder bleiben ansprechbar und können die Tiefe der Analgesie über die Atmung selbst steuern. Weil Nüchternheit nicht erforderlich ist, ist die Anwendung sowohl notfallmäßig als auch elektiv möglich. In der Kinderchirurgie der Universitätsklinik Mannheim setzen Dr. Bettina Lange und Kollegen LIVOPAN ${ }^{\circledR}$ seit August 2010 ein - etwa in der Wundversorgung und bei Verbandswechseln, Luxationen und leichten Frakturen, Fremdkörper- und Metallentfernung, leichten Verbrennungen, Abszessinzisionen oder Punktionen.„Bei solchen kleineren Eingriffen kann mit LIVOPAN ${ }^{\circledR}$ eine Vollnarkose umgangen werden", sagte Lange. Bei 149 Kindern im mittleren Alter von 8,5 Jahren angewendet war die Wirkung in $98 \%$ der Fälle gut, in $97 \%$ ohne Nebenwirkungen.

In der interdisziplinären Kindernotaufnahme in Karlsruhe, die jährlich 29.000 Patienten kinderchirurgisch und pädiatrisch versorgt, wird das Gasgemisch seit Ende 2009 eingesetzt und als schnelle, effektive und praktikable Schmerztherapie geschätzt, wie Leiter Dr. Matthias Kuch berichtete: Schon nach kurzer Zeit sei LIVOPAN ${ }^{\circledR}$ aus dem klinischen Alltag nicht mehr wegzudenken gewesen.

Michael Koczorek

Symposium „LIVOPAN ${ }^{\circledR}$. Erfahrungsberichte zur inhalativen Analgesie mit einer äquimolaren Fertigmischung aus Sauerstoff und Lachgas". 107. Jahrestagung der DGKJ. Bielefeld, 24. September 2011. Veranstalter: Linde Gas Therapeutics

Gens. Durch diese Form der Resistenz tritt der Knock-Down zwar verzögert ein, dieie gleichmäßige sowie ausreichend lange und intensive Benetzung der Haare sorgt aber dafür, dass selbst mobile Läuse kontinuierlich einer letalen Wirkstoffdosis ausgesetzt sind. Darüber hinaus beeinflussen formulierungsspezifische Merkmale die Wirksamkeit. Pyrethrum z. B. ist in Arzneimittelform (Goldgeist forte) mit dem bewährten Resistenzbrecher und Synergisten Piperonylbutoxid kombiniert. So wird die Wirksamkeit um das 30-Fache verstärkt und die Resistenzbildung verhindert.

Nach Informationen von EDUARD GERLACH 